\title{
Commemorating Britton Chance
}

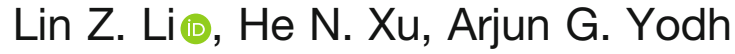 \\ University of Pennsylvania, Philadelphia, USA
}

Britton Chance excelled in research, at a remarkably high level, in areas spanning biochemistry, bioengineering, biology, biophysics, chemical enginerring, electrical engineering, medicine, and physics [1]. He was a pioneer in bioenergetics, redox biology, and in vivo NMR, and was also a founding father of in vivo biomedical optics. He also led the development of radar system in the Radiation Laboratory of MIT during the World War II. At the bench, Chance measured the enzymatic kinetics, experimentally demonstrated the formation of the MichaelisMenten enzyme-substrate complex, and he and coworkers identified the release of hydrogen peroxide by mitochondrial respiratory chain. Moreover, he was an experimental pioneer in the study and elucidation of electron-tunneling phenomena in biological systems. Throughout the time, he and his coworkers and colleagues invented many of the instruments and techniques that are widely utilized to probe biology at sub-cellular, cellular, tissue, and organ levels. Lastly, he was a leader in the translation of basic technologies to diagnostic and treatment strategies in the clinic. Among these instruments and technologies are the dualwavelength spectrometer, miniature stop-flow devices, the redox scanner, in vivo ${ }^{31} \mathrm{P}-\mathrm{MRS}$, time-resolved near-infrared spectroscopy (NIRS), photon diffusion tomography, and functional NIRS. Additionally, some of his early work included the development of a mechanical computer for solving differential equations, and the essential input for the construction of logical circuits of the ENIAC, the first general purpose electronic computer. During his life, Chance was awarded many honors including the US National Medal of Science and memberships in six Academies of Science, but perhaps his 1952 Olympic gold medal in sailing was his most legendary award. He also trained many students and researchers all over the world and thus helped build today's global research community that emphasizes research innovation, biomedical application, and clinical translation of new technologies.

To celebrate his life, time, and legacy, several symposia were organized and successfully held at the University of Pennsylvania, where Chance spent nearly eight decades of his research career. These symposia were the Britton Chance

Correspondence to: Lin Li; e-mail: linli@pennmedicine.upenn.edu
Memorial Symposium (2011), the Britton Chance Centennial Symposium on Metabolic Imaging and Spectroscopy (2013), and the Second Britton Chance International Symposium on Metabolic Imaging and Spectroscopy (2018) celebrating his 105th birthday. To honor his contributions and carry his scientific torch further, hereafter, we will organize a Britton Chance Symposium every 5 years.

The 2018 Chance symposium, held June 11-13, 2018, attracted over 200 participants from North America, Europe, and Asia. The symposium featured approximately 100 abstracts, 48 invited and contributed talks, and a panel discussion concerning critical issues in the field of metabolic imaging and spectroscopy. The speakers included leading scientists, engineers, and clinicians. Eight keynote speakers discussed frontier topics in cancer metabolic plasticity, molecular imaging of cancer metabolism and cancer theranostics, imaging of pancreatic islets, redox biology, stimulated Raman scattering microscopy, radiation therapy, and magnetic resonance spectroscopy studies of mitochondrial neuroenergetics underlying brain function. Twenty-four students, postdoctoral fellows, and early stage young investigators received stipends to attend the symposium and present their research work. The conference program, photos, video presentations, and more can be found at the web site: https://www.med.upenn.edu/chance.

Working in concert with the Journal of Biomedical Optics (JBO) and Molecular Imaging Biology (MIB), the Organizing Committee solicited manuscript contributions from 2018 Symposium participants for peer reviewed publication. These articles aim, in part, to commemorate the 2018 symposium. Thirteen of these articles are included in JBO volume 24 issue 5, and four of these articles are included in this issue of MIB.

In particular, and briefly, the article entitled "Optical redox imaging detects the effects of DEK oncogene knockdown on the redox state of MDA-MB-231 breast cancer cells", by Wen et al., presents experimental evidence that oncogene suppression leads to changes in optical redox imaging of intrinsic NADH and FAD fluorescence that are concurrent with a reduction in the invasive potential of an aggressive breast cancer cell line [2]. The article entitled "Optical redox imaging of fixed unstained muscle slides reveals useful biological information", by Xu et al., quantifies age-associated redox differences by imaging 
autofluorescence (across the same excitation and emission channels as used for NADH and FAD) of formalin fixed muscle slides [3]. Two other articles are by Ramanujan et al. [4], and $\mathrm{Xu}$ et al. [5], respectively. In these works, fluorescence imaging techniques were applied to investigate the treatment response of mammary tumor spheroids, and melanoma cells and xenografts, respectively. Both manuscripts demonstrated significant intratumor heterogeneity that may be modified by chemotherapy. The first paper employs two-photon imaging of exogenous dyes sensitive to cell viability and metabolism, while the latter paper utilizes optical redox imaging of NADH and FAD. Britton Chance extensively utilized the intrinsic fluorescence of mitochondrial NADH and oxidized flavoproteins containing FAD as the basis of optical redox imaging. Privately, he regarded this as "perhaps the most important discovery" of his career because "for the first time, we could obtain optical signals from living mitochondrial tissues" and it "opened a new field of metabolic research" [1]. On the other hand, to understand the metabolic and functional activities in cells and tissues more specifically and completely, exogenous dyes and sensors are also great tools (Thanks, in part, to the rapid development and use of exogenous dyes over the past few decades). The article by Ramanujan et al., for example, presents an excellent example of how multiple exogenous fluorescent dyes can be utilized to provide an integrated picture of spheroid viability and metabolism that respond differently to several chemotherapies. In addition to the articles published in this issue demonstrating the value of fluorescence imaging and optical redox imaging, we note that new developments in biomedical optics such as photoacoustic tomography, stimulated Raman scattering microscopy, fluorescence life-time microscopy of NADH and FAD, functional NIRS, high resolution neuron network mapping, Cherenkov luminescence imaging were also covered in the 2013 and 2018 Chance symposia.

To conclude, we thank all authors, reviewers, editors, and staff who supported the symposium and the special journal issues. Of course, we thank Britton Chance too, for continued inspiration.

\section{Compliance with Ethical Standards}

\section{Conflict of Interest}

The authors declare that they have no conflicts of interest.

\section{References}

1. Li LZ, Leydesdorff L, Sun N, Nioka S, Garfield E (2014) Citation analysis of the scientific publications of Britton Chance in ISI citation indexes. J Innov Opt Health Sci 07:1430003

2. Wen Y, Xu HN, Privette Vinnedge LM et al (2019) Optical redox imaging detects the effects of DEK oncogene knockdown on the redox state of MDA-MB-231 breast cancer cells. Mol Imaging Biol 20. https://doi.org/10.1007/s11307-019-01321-w

3. Xu HN, Zhao H, Chellappa K et al (2019) Optical redox imaging of fixed unstained muscle slides reveals useful biological information. Mol Imaging Biol 20. https://doi.org/10.1007/s11307-019-01348-z

4. Ramanujan VK (2019) Quantitative imaging of morphometric and metabolic signatures reveals heterogeneity in drug response of threedimensional mammary tumor spheroids. Mol Imaging Biol 20. https:// doi.org/10.1007/s11307-019-01324-7

5. Xu HN, Feng M, Nath K, Nelson D, Roman J, Zhao H, Lin Z, Glickson JD, Li LZ (2019) Optical redox imaging of lonidamine treatment response of melanoma cells and xenografts. Mol Imaging Biol 20. https://doi.org/10.1007/s11307-018-1258-z

Publisher's Note Springer Nature remains neutral with regard to jurisdictional claims in published maps and institutional affiliations. 